\title{
Scientific Methodological Approach to Effective Contract Assessment
}

\author{
Yulia Romanova \\ Management Department \\ Financial University under the Government of the Russian \\ Federation \\ Moscow, Russia \\ E-mail: ryulia1@yandex.ru
}

\author{
Anna Egorenko \\ Management Department \\ Financial University under the Government of the Russian \\ Federation \\ Moscow, Russia \\ E-mail: annaegorenko@yandex.ru
}

\begin{abstract}
The article is devoted to questions of scientific methodological approach for the evaluation of contract effectiveness introduced in higher education, in particular, the introduction of new forms of labor remuneration and the transfer of the academic teaching staff to a new management technology. The article analyzes the value of teachers' motivation to work efficiency, professional competence development, and obtaining scientific results. The main attention is paid to the materials of the Ministry of Education and Science of the Russian Federation, focused on the importance of work indicators record, scientific publications, cited articles, employment in the educational sector, and others. Under the current conditions there are rules and regulations for the use of an effective contract in the education system. Nevertheless, the matter of assessing professional performance of academic teaching staff in terms of effectiveness is still highly relevant and insufficiently developed in the scientific literature.
\end{abstract}

Keywords-scientific work; an effective contract; human resources

\section{INTRODUCTION}

The education system in modern conditions plays an increasingly important role as it is the only system capable of fulfilling a most complex demand of today's society: to form and maintain the brainpower of a nation and to develop and improve new technologies. Both these processes are highly interconnected and interdependent. National brainpower consists in those intellectual forces which are capable of pushing a country to a qualitatively new state. On the other hand, these forces have vast intellectual resources, the necessary technological knowledge and thinking, skills and ability to constantly grow.

The education system has a unique opportunity to influence the formation of specific patterns of thinking and behavior that are able to develop and promote new technological principles and technologies not only in individual industries, but also in a country as a whole. It is increasingly becoming a major factor of economic development.

Russian authors whose works are devoted to the efficient contract evaluation and the education system as a whole include: S. Bagautdinova, N. Levshina, L Sannikov, S.Yurevich [1], Y. Gnezdovoy [4,5, 9], V. Tambovtsev [7], A. Tseluyko [8], and others. In addition, the problems of higher education management and teachers' motivation are reflected in the works of such foreign authors as J. Bess [2] W. Brown Jr. [3], R. McCormick and R. Meiners [6].

The global technology race cannot be won without the optimal participation of the national education system. Modern experts say that the aging of professional and technological knowledge, skills, and abilities occurs in 1.5 years, and this process is constantly speeding up. Therefore, they need to be constantly upgraded and improved. This fact makes completely new conditions and challenges for the entire education system.

An important aspect of an education system's functionality is the fact that it reconstructs and modifies culture as a set of values, convictions and norms of behavior, which contributes to preserving the stableness of society and also to its development. Under the circumstances, finance and intelligence, which are the main resources of development in the society of information, are extremely exposed to world globalization and are losing territorial binding, constantly moving between countries and regions, the functioning of education is paramount

In a rapidly changing socio-economic environment, professional human adaptation problems are intensified, which leads to greater competition between different educational systems.

In this context, the problem of assessing the potential of Russian educational institutions and that of an effective contract are regarded as the most important problems of the Russian educational system.

\section{OBJECTIVES AND METHODS}

The term "potential" means "hidden features, power, and strength". The broad interpretation of the semantic notion of "potential" consists in its consideration as "a source of opportunities, resources, reserve that can be activated, used to solve a problem or achieve a specific purpose; the capacity of an individual, society or state in a particular area" [1]. 
Thus, the term "potential" means having some hidden capacity or ability in a certain sphere, which have not been shown yet.

It should be noted that there are various meanings of the concept "potential" in respect of the society's socioeconomic development:

- the multi-faceted approach: potential is viewed as a unity of different resources, mainly economic, directly related to the operation of production and the acceleration of scientific and technical progress, necessary for the successful operation and/or development of the system.

- the systematic approach: potential is a system of different (material, labor) factors that allow the society to reach its goals (in production).

- the resource approach: potential as an opportunity of a set of resources to solve complex tasks. Thus, the more rational the structure of the object is, the better its structural and functional elements are correlated, the greater its capacity and efficiency is.

Scientific potential is a real capacity of a society at its disposal for research and application of research results in social practice. The problem in its studying was caused primarily by the urgent tasks of management science, such as improving its efficiency and the need for practical implementation of these results.

The solution to this problem is impossible without setting and solving the task of quantitative evaluation of scientific potential and structuring its components.

According to the definition of UNESCO, scientific potential is the total of disposable resources of a country for scientific discoveries, inventions, and technological innovations, as well as for addressing national and international problems posed by science and its applications [6].

Scientific potential's, as a social practice phenomenon, essence and its impact are studied by economics. Within the framework of this science, specific empirical material has been studied and analyzed with the help of quantitative measurement methods of individual parameters. Despite the significance of this study, it should be noted that not all the components of the given phenomenon find their quantitative display, for example, currently available scientific knowledge, organizational factors of the science, etc.

\section{RESULTS AND DISCUSSION}

\section{A. Management of Human Resources in the Education System in the Context of Organizational Changes}

Over the past two decades the increasing role of education as a social and economic phenomenon can be traced quite considerably, this process is actively involved in the life of every civilized state. Improving the education system has become one of the main priorities of the longterm strategy of social and economic policy in the development of society. This phenomenon exists both at the personal level (at the level of each individual) and at the social level. Being one of the main means of obtaining knowledge and developing human intellectual abilities, education allows to actively participate in the political, economic and cultural life of the entire world community, which enables the society and the nation as a whole to strengthen its intellectual and political independence, as well as to participate in international life, in particular, to preserve, enrich, and spread global and national culture.

Currently, the matters of education and development of human intellectual abilities, including investment in human resources, are becoming paramount.

Over the past two decades, the Russian education system has undergone fundamental changes. The system has developed the essential characteristics that affect all the processes which take place in higher education. This is, above all, the emergence and fairly rapid formation of the non-state sector of education that has significantly expanded the organizational and functional freedom of the country's educational sphere and allowed various types of educational institutions to operate in the same field (public and private, commercial and non-commercial). The development and implementation of educational curriculums by various unified programs has been an important step in reforming the education system. These curriculums comprise the federal, regional, and local components. The inevitable consequence of this process is the creation and development of market management mechanisms and educational institutions funding. The current state of higher education urgently requires a gradual transition to a multi-level training system for students of higher qualification.

The processes taking place in the education system are ambiguous; they require in-depth analysis and comprehensive evaluation, but it is already possible to note the increased democracy of the education system [7].

In general, it can be said that the education system has become more democratic and responsive to labor market demands. Moreover, the global trend of education of adults, their training, retraining, and qualification upgrading is clearly seen. The educational potential of the country is significantly expanding not only by quantitative, but also by qualitative modifications of educational institutions (the growth of their numbers, the appearance of the branches of capital high schools in small towns, the introduction and development of modern information technology), which leads to serious stratification shifts. The impact of education as a social institution on the modern society is increasing steadily [8].

In this regard, we can say that the system of Russian education, including higher education, is undergoing global changes, which include organizational changes.

\section{B. Assessment of the Scientific Potential in Terms of Effective Contract Implementation}

Like any system with a high degree of complexity, scientific potential cannot be represented as a sum of elementary quantitative measurements of its components, it 
gets a new quality as a result of their interaction and mutual influence.

Direct counting and extrapolations give characteristic of the lowest, simplest level of the phenomenon under investigation. Complex, mostly qualitative parameters characterize the patterns of development of the higher specific organization levels of the science. Thus, it is possible to obtain full and corresponding to reality characteristics of the scientific potential only by using the qualitative and quantitative analysis altogether [9].

This fact accounts for the difficulties that arise while evaluating scientific potential in the whole country, both in a separate branch of science and in a particular higher education institution or scientific organization, besides, it presupposes the integral character of studying. A lot of works of prominent Russian scientists have been dedicated to solving this problem, some of them were written in the Soviet period.

Unfortunately, the focus of research has been directed to the study of scientific capacity and effectiveness of the scientific research institutes, design bureaus, etc., which work in the mode "development - implementation". Essentially, they recognized some secondary nature of science in high school compared to training activities; the problems of scientific potential were almost out of sight of both scientists and administrative staff. Scientific potential used to be evaluated like this: "There is some research work done, there is a certain result, which is sufficient". However, it is obvious that scientific potential is a significant factor in the functioning and development of higher education science, and, therefore, the scientific work of its staff is very specific, requiring different evaluation parameters from those used in assessing the performance of the employees of a research institute or a design engineering bureau.

We should also bear in mind the fact that the scientific potential of a university is created not only by its specialists, but also by its students, postgraduate, and candidates.

The evaluation of a scientist's labor effectiveness forms the scientific potential of the organization as a whole. It should be kept in mind that the efficiency of research activities is defined by numerous components (partial indicators), each of which has a certain importance and weight.

We have to admit that in the evaluation of universities research work the intuitive approach is currently dominating. It was developed by administrative structures on the basis of knowledge and experience, which replaced scientifically developed and validated methods. As a consequence of this approach, there is not always a scientifically motivated and objective evaluation of the professional activity of higher education specialists, performed not only by individual experts, but also the relevant administrative services.

With regard to the conditions of higher school activities, it would be rational to speak of its scientific and educational potential as both sides of such activity constitute interrelated and interconnected components. At a certain stage and under certain conditions it is possible to distinguish one of the sides of this activity, however, in general, it is necessary to take into account the dialectical relationship of educational and scientific processes.

An effective contract can be a tool for raising the scientific and educational potential of not only individual universities, but also the education system as a whole, also it can serve as an evaluation method [4].

To assess the contribution of employees to the scientific and educational potential of a university and the development of an effective contract, the basic components must be clearly established. They can be considered as the main directions of an effective contract in the scientific part of university employees' professional activity.

What made it possible to define the following components of the scientific component:

- independent research activity (preparation of articles, monographs, theses, etc.);

- scientific supervision of students, postgraduates, interaction with student research organizations;

- management activities in the field of science (including fundraising from external customers of scientific and technical products).

The following items allow to establish evaluation criteria of contribution of a staff member to the scientific and educational potential of a university:

- the position held in the high school science management system;

- the number of published scientific articles during the reporting period;

- scientific significance of such articles;

- participation in state budgetary research conducted at the university;

- the amount of funds allocated on research by external customers (out of budgetary organizations);

- candidate and doctoral theses presentation, obtaining academic degrees;

- the number of graduate students, doctoral students and applicants who defended their thesis for a degree under the supervision of an employee;

- the proportion of graduate students (applicants) who defended their thesis under the supervision of an employee and went on working in their university;

- the proportion of students who defended their final qualifying works under the supervision of an employee and entered the graduate school;

- the number of students who prepared their scientific articles and presentations for scientific conferences under the guidance of a scientific employee;

- the number of students who prepared their scientific works which won 
university/city/regional/national/international competitions under the guidance of a scientific worker;

- the number of students who won grants for research and development work and took part in thematic research projects under the guidance of a university employee;

- the degree of employee participation in the activities of student scientific organizations of the university.

Naturally, a proper assessment unit is needed for the objectivity of evaluative judgments. This will boil down all the results to a single performance criterion. Experience has shown that the most rational option is using the points-based system (scale). This will eliminate the possible subjectivity of such an assessment.

Taking into account the specific character of the scientific potential structure of higher education, it is advisable to use these indicators (in adapted form) in an effective contract not only of the teaching staff, but also of graduate students, students and all the employees who fall within the scope of such a contract. The significance of students' research work and the necessity to increase the creative component of scientific potential account for a large number of indicators related to the scientific supervision of graduate students.

Only a well-organized system of students' research work in the university can solve the important task of making students creative individuals, who are able to effectively deal with problems connected with production or other issues caused by the modern society. Practice shows that creative abilities of graduates, their involvement in science, ability to work with scientific progress at the present stage are not a less important component of professionalism than their theoretical knowledge [2].

The current ongoing process of creating an integrated research and education sector, which uses the science and higher education potential of Russian science, is objectively needed. It should be a set of leading scientific, research and educational institutions with the appropriate status, and support from state, private and other institutions. This process is developing in several relatively independent directions.

The experience gained abroad and in our country shows that such an integration involves the creation of complex innovation business organizations around major scientific and educational centers. Such organizations include science and technology parks, business incubators, engineering centers, etc. It is very important that this process in Russia must not be artificially forced. On the contrary, it should be maintained as part of a special program, designed in accordance with the development of the market and innovation activities, broken into several logical steps. One of the tools of this process should be the introduction of educational contracts in the field of science and practice (including in the field of university pedagogy), including effective contracts. It should fully meet the needs of society for specialists.

The educational contract as a scientific potential recovery tool is a complex phenomenon. A comprehensive assessment of the scientific and educational potential of the university involves distinguishing the component of assessing the teaching staff as an independent and very important component of scientific potential.

In Russia, the only way to assess the human resource capacity of the university is accreditation [3]. Let us consider the principles of personnel potential assessment via accreditation:

1. The criteria are divided into two groups: basic and socalled background. The final integrated assessment is based on the basic (priority) criteria.

2. They assess not only individual qualification groups, but also the balance of the whole structure. Special attention is paid to university management and academic leaders (for example, heads of research schools). used:

3 . In the course of accreditation two types of criteria are

- preservation of human resource capacity;

- dynamics of development (trends in structural changes, orientation of the teaching staff to professional development, coherence and prospect of the personnel policy).

The leading assessments of the second type:

4. Not only the very subject of scientific and educational activities, but also prerequisites for effective scientific pedagogical activity (conditions for scientific communication, time budget, etc.), and also cognitive, intellectual, social and organizational aspects of the activity are subject to evaluation.

5. Transfer of focus from evaluation of public certification indicators (degree, rank) on the cognitive and social indicators: assessment of the cognitive contents of a scientific product (solidity, innovation, impact on other sectors, practical impact).

6. Evaluation of the motivations (and psychological characteristics) of scientific pedagogical workers.

7. Methodological qualities are separately assessed. In accredited institutions of higher education training should not be combined with research - it should be performed through research.

8. Evaluation of information literacy: the knowledge of the "leading edge" of the world and domestic science, including in cross-country scientific communication.

9. Topical and social professional mobility.

It must be noted that, in Russia, there is still no single methodology for assessing the human resource potential of a university. As an option, human resources of a university can 
be represented as a sum of human potentials of employees of the university [5].

The main directions of scientific activity of university employees are the basis for the assessment of their contribution to human resources potential. This allows to determine its essential components.

The existing approaches to evaluating the scientific potential of the educational institutions have, in our opinion, the same disadvantage. They are based on an assessment of a separate employee's contribution according to the results of performed activities. However, we would like to go back to the definition of potential as "a source of capacities, resources, reserves, which can be powered by ...". On this basis, we can say that the urgent task is the development of techniques to identify hidden capacities of university teaching and research staff, to understand what these workers are capable of. Such techniques are individual and difficult to formalize. A special atmosphere must be created at universities so that employees have an opportunity to reveal their scientific potential. Under these circumstances, the role of university management becomes more important. The personality of the university head is the key element of the functioning of the institution as a whole. University administration is responsible:

for the development of university strategy;

for the selection of teaching staff;

for the creation of a strong team;

for the promotion of the university in the educational space;

for searching for additional sources of funding.

\section{CONCLUSIONS AND RECOMMENDATIONS}

Using the scientific potential of university employees requires coordinated work of the whole team. A team is the most common form of innovative communities. Teamwork requires many qualities of employees, which cannot be shown in individual work. These qualities raise scientific workers to a new level of professional, scientific and creative potential, accelerate the development of individual employees and the organization as a whole.

Global trends in science development influence the scientific potential of universities. The scientific future is for those states that have well-developed science and education. However, it is possible to actually compare the levels of scientific and educational potential only relatively as it is impossible to measure the quality of knowledge with the help of a physical unit.

The main problem in Russia today is the process of "brain drain" to external markets and change of scientists' activity, their shift to commercial spheres. Therefore, we can establish the fact that the scientific potential of our country is relatively high, but, unfortunately, it has negative dynamics.

\section{REFERENCES}

[1] S. Bagautdinova, N. Levshina, L. Sannikov, S. Yurevich. Effective Contract as a Means of Assessing the Activity of a Preschool Education Teacher // Modern High Technologies. - 2016. - № 2-2. pp. 289-293

[2] J. Bess. Contracts, the Bureaucracy and Motivation of Teachers: Possible Effects of Lack of Permanent Employment Contracts. Contracts in the Academic World. Comp. and scientific ed. M. Yudkevich. Moscow: Publishing House of High Economic School. 2011.

[3] W. Brown Jr. University Management and a Permanent Recruitment Contract in Academic Environment: Study in terms of Property Rights. Contracts in the Academic World. Comp. and scientific. ed. M Yudkevich. Moscow: Publishing House of High Economic School. 2011.

[4] Y. Gnezdova. Educational Outsourcing - the Innovational Technology of Attracting External Resources in the Public Sector / Y. Gnezdova // Innovational Technology in Modern Education Based on the materials from the Third International Scientific and Practical Internet-conference. 2016. pp 137-141.

[5] Y. Gnezdova. The Specific Character of the Application of the Educational Outsourcing Organizational Model of / Y. Gnezdova // Educational Technology (Moscow). 2016. № 2. pp. 73-78.

[6] R. McCormick and R. Meiners. University Management: A View from the Perspective of Property Rights. Contracts in the Academic World. Comp. and scientific. ed. M. Yudkevich. Moscow: Publishing House of High Economic School. 2011.

[7] V. Tambovtsev. Outcomes of the Education Industry and the Problems of the Measurement of Educational Technologies. Education Matters. 2008, № 1.

[8] A. Tseluyko. Transformation of Organizational Knowledge in Complex Environment. // Economics \& Management: Problems and Solutions. - 2015, №8. - pp.98-106.

[9] Y. Gnezdova. Modern aspects of the development of interneteconomy in Russia / Y. Gnezdova, Y. Chernyavskaya, L. Rubtsova, N. Soldatova, I. Idilov // Journal of Internet Banking and Commerce. 2016. T. 21. № SPEC.ISSUE 4 